\title{
Desarrollo territorial rural y cooperativas: un análisis desde las políticas públicas
}

Inmaculada Buendía-Martínez*

\author{
Alain Côté \\ Recibido: 2014-02-I8 Aprobado: 2014-05-I4 Disponible en línea: 20I4-07-27 \\ doi:I0.III44/javeriana.CRDII-74.dtrc
}

Cómo citar este artículo: Buendía-Martínez, I. y Côté, A. (2014). Desarrollo territorial rural y cooperativas: un análisis desde las políticas públicas. Cuadernos de Desarrollo Rural, II(74), 35-54. http://dx.doi.org/I0.III44/Javeriana. CRDII-74.dtrc

\section{Resumen}

Las zonas rurales enfrentan un conjunto de desafíos globales como resultado de las actuales transformaciones económicas y sociales. La contribución de las cooperativas, en tanto que empresas de estructura colectiva con una fuerte adscripción territorial, ha originado que sean consideradas en algunos países como un pilar de las políticas rurales. Este trabajo analiza una exitosa iniciativa pública de casi treinta años: las cooperativas de desarrollo regional. Su función como promotoras del movimiento cooperativo de Quebec es fundamental para la diversidad empresarial y el empleo de las regiones rurales.

\section{Palabras clave:}

cooperativas; desarrollo territorial rural; política rural; cooperativa de desarrollo regional; políticas públicas

\footnotetext{
*Doctora en Ciencias Económicas y Empresariales. Profesora del área de Política Económica de la Universidad de Castilla-La Mancha (España).Correo electrónico: inmaculada.buendia@uclm.es ** Licenciado en Ciencias Económicas por la Universidad de Montreal (Canadá) y Master en Desarrollo Regional por la Universidad de Quebec en Rimouski (Canadá). Director adjunto del Centro de Estudios Desjardins en Gestión de Cooperativas de Servicios Financieros de HEC Montreal (Canadá).

Correo electrónico: cote.alain@hec.ca
}

\section{cc) 0}




\title{
Territorial Rural Development and Cooperatives: an Analysis from Public Policies
}

\begin{abstract}
Rural areas face a range of global challenges as a result of current economic and social transformations. The contribution of cooperatives, as collective companies with a strong territorial affiliation, has led them to be considered in some countries as a pillar of rural policies. This paper analyzes a successful public initiative released almost thirty years ago: cooperatives for regional development. Their function as promoters of the cooperative movement in Quebec is critical for corporate diversity and employment in rural areas.
\end{abstract}

\section{Keywords:}

cooperatives; rural territorial development; rural policy; cooperatives for regional development; public policies.

\section{Développement territorial rural et les Coopératives: une analyse dès les politiques publiques}

\section{Résumé}

Les zones rurales affrontent un ensemble de défis globaux comme résultat des nouvelles transformations économiques et sociales. La contribution des coopératives, en étant elles-mêmes des entreprises de structure collective avec une forte compétence territoriale, a occasionné quélles soient considérées, dans quelques pays, comme un pilier des politiques rurales. Ce travail analyse une initiative publique à succès de presque 30 ans: les coopératives de développement régional. Leur fonction comme promotrices du mouvement coopératif québécois est essentiel pour la diversité entrepreneuriale et l’emploi des régions rurales.

Mots-clés:

coopératives; développement territorial rural; politique rurale; coopérative de développement régional; politiques publiques. 


\section{Introducción}

La globalización de la economía es, probablemente, el factor más aludido en las tres últimas décadas como causa del cambio en el entorno económico. Este nuevo escenario, junto con la proliferación de las nuevas tecnologías, la investigación agraria y la modificación en los hábitos de los consumidores, está generando un proceso de transformación de las economías rurales cada vez menos dependientes de las actividades agrícolas, con el consiguiente impacto negativo en los ingresos agrarios y en las oportunidades de trabajo (Organización para la Cooperación y Desarrollo Económicos [OCDE], 1993).

A estas mutaciones se añaden los problemas de despoblamiento, envejecimiento de la población, falta de cualificaciones y baja productividad de la mano de obra, lo que trae como consecuencia una continua pérdida de masa crítica. Esto hace inviable el mantenimiento de los servicios públicos y de las infraestructuras, y limita el desarrollo empresarial (OCDE, 2006). En definitiva, se podría afirmar que las áreas rurales están ante un proceso de desrruralización, definido no solo por los cambios en los contenidos de la propia definición de lo rural, sino también por la pérdida de importancia cuantitativa respecto del ámbito urbano (García Sanz, 1997, p. 35).

Estas dinámicas requieren de nuevos enfoques políticos para encarar los desafíos del mundo rural. Desde el comienzo del nuevo siglo y en el entorno de la OCDE, se ha producido una reorientación en la intervención de los poderes públicos en las áreas rurales, lo que significa una evolución desde las políticas sectoriales, cuyo objetivo era la reconversión del sector agrario basada en apoyos financieros a otras políticas en que era necesario poner en valor las ventajas comparativas de los territorios (Pezzini, 2003).

El desarrollo rural requiere de una política proactiva basada en un enfoque integrador que consiga el equilibrio entre los sectores económicos, las administraciones públicas implicadas y los agentes públicos y privados (OCDE, 2006). Desde una perspectiva económica, los diferentes enfoques coinciden en considerar la actividad emprendedora como una intervención estratégica con un efecto acelerador de los procesos de desarrollo rural (Organización de las Naciones Unidas para la Alimentación y la Agricultura [FAO, por sus siglas en inglés], 1997). Pero la generación de empresariado en las zonas rurales no está exenta de dificultades, lo que es consecuencia tanto de las particularidades de la población rural como de las limitaciones del entorno empresarial. Esta heterogeneidad requiere diferentes intervenciones públicas entre las que se encuentran: el fomento y apoyo a los nuevos emprendimientos, la promoción de la cultura empresarial, la educación 
empresarial y el soporte para el crecimiento, la sobrevivencia y el aumento de competitividad de las Pymes existentes (North y Smallbone, 2006).

Dentro de los casos exitosos de intervenciones públicas en el ámbito rural está la experiencia quebequense. Considerada como una de las más avanzadas de la OCDE, su política rural está basada en conseguir la autonomía de las colectividades y la ocupación del territorio sin especificidades sectoriales (OCDE, 20I0). La superficie rural de Quebec es superior a 90\% de su territorio, el mayor de todas las provincias canadienses, y representa 20\% del Producto Interior Bruto (PIB) provincial, con un aumento de la población y de los ingresos de los hogares y con un tejido empresarial en continua diversificación. El análisis de este último factor revela la importancia económica que el movimiento cooperativo tiene en la provincia, el cual ha registrado una consolidación y fortalecimiento desde la década de los años ochenta, gracias una ambiciosa política de desarrollo cooperativo (Arteau, Brassard y Malo, 2005; Girard y Brière, I999; Ministère des Finances, de l’Économie et de la Recherche, 2003).

Si bien la idoneidad de la fórmula cooperativa en los procesos de desarrollo territorial rural está documentada en la literatura, su análisis desde la perspectiva de las políticas públicas es escasa (Chaves, 20I2). Con base en ello, el trabajo plantea como problemática la relación entre la política de empresa de desarrollo cooperativo y la política rural; es decir, si las medidas para el fomento de cooperativas favorecen el incremento sostenido de los niveles de vida de la población rural y apoya, al mismo, tiempo el crecimiento económico nacional.

Limitando el análisis a aquellas políticas públicas de corte económico, entendiendo como tales la intervención deliberada de los poderes públicos en la actividad económica, este trabajo pretende analizar la vinculación de las empresas cooperativas en el desarrollo rural. Para esto se ha utilizado un diseño investigativo de tipo descriptivo que, en una primera etapa, se ha centrado en revisar la literatura sobre la relación entre cooperativas y desarrollo rural.

En una segunda fase, se ha procedido a escudriñar los programas, iniciativas y medidas públicas centradas en cooperativismo y desarrollo rural en el ámbito nacional canadiense y provincial quebequense. El análisis concluye con el estudio de uno de los instrumentos clave de la política de desarrollo cooperativo quebequense: las cooperativas de desarrollo regional (CDR) que, con casi tres décadas en funcionamiento, representan una iniciativa pública exitosa de generación colectiva, con importantes efectos en la diversidad empresarial y en el empleo de las regiones rurales de Quebec. 


\section{Cooperativas: enfoque empresarial estratégico del desarrollo rural}

La complejidad del mundo rural y la incertidumbre sobre el impacto de muchos de los cambios vinculados con la demografía, la economía y el medioambiente genera posiciones heterogéneas entre los expertos y los responsables políticos en torno a los modelos y las formas de intervención. A pesar de ello, existe unanimidad entre los investigadores acerca de los rasgos que caracterizan la actual realidad rural, destacando, entre otros: la importancia de la dimensión territorial; la vinculación entre los ámbitos urbano y rural; la complementariedad de las actividades agrarias y aquellas no agrícolas; la integración del mundo rural en los mercados nacionales e internacionales; y la protección del medio ambiente (Grammont, 2008; Unidad Regional de Desarrollo Agrícola y Rural Sostenible [Lcses, por sus siglas en inglés], 2003, p. 9; OCDE, 2006; Ruiz Rivera y Delgado Campos, 2008). Este nuevo escenario implica que el desarrollo rural debe mejorar no solo las oportunidades económicas, sino también debe conseguir el bienestar de la población y de la protección de las tradiciones socioculturales; esto involucra el diseño de procesos integrales, dinámicos y complejos, que susciten la participación de diferentes sectores de la población (Coque Martínez, 2005).

En este contexto, la actividad emprendedora es el vehículo para mejorar la calidad de vida de la población y para hacer sostenible la economía y el medio ambiente de las comunidades rurales. La orientación del empresariado en las áreas rurales ha de estar basada en la estimulación del talento empresarial local con el consiguiente crecimiento de empresas autóctonas. De esta forma se generaría empleo y valor económico regional, y también los recursos se mantendrían en el territorio (Fuller-Love, Midmore, Thomas y Henley, 2006; Petrin, 1997).

Dentro del amplio abanico de fórmulas jurídicas que se pueden utilizar para lograr la reactivación de las áreas rurales, las cooperativas resultan un mecanismo apropiado para asegurar un desarrollo integrado (Comunidades Europeas, 1988), dado que permiten aprovechar los recursos autóctonos al fomentar la cohesión regional, contribuir al desarrollo de comportamientos democráticos y reforzar el interés de la colectividad. Este aspecto resulta de especial importancia en áreas rurales con un perfil económico débil, donde su contribución es decisiva para la generación y el mantenimiento de empleo, de infraestructuras socioeconómicas diversas y de actividades encuadradas en los sectores secundario y terciario. 
De esta manera, los beneficios se reinvierten en los lugares de origen, lo que evita el éxodo de la población (Comunidades Europeas, 1987). Además, las cooperativas ofrecen una base racional para el desarrollo económico y social de carácter participativo: facilita la concertación económica y social mediante la generación de una demanda organizada de bienes o servicios, donde pueden manifestarse las necesidades y preferencias de la población (Instituto de Promoción de la Economía Social [IPES], 1996, p. 48).

Esta oportunidad que representan las cooperativas para el desarrollo rural tiene su base en su configuración organizativa, en tanto estructura de movilización colectiva con un fuerte componente de capital social (OCDE, 20ro). En efecto, la concepción de cooperativa como asociación para emprender de otra manera, implica una acción colectiva fundamentada en tres dimensiones: la social (asociarse), la económica (emprender) y la política (de modo distinto) (Favreau, 2009, p. 7). De esta forma, la viabilidad y la supervivencia de las cooperativas dependen de su capacidad para convertir en actividades económicas las especificidades vinculadas al territorio, en tanto que empresa ciudadana, asociativa y participativa (Arteau y Brassard, 2008, p. II).

Esta acción colectiva configura las cooperativas como organizaciones con un fuerte componente de capital social (Valentinov, 2004). Considerado como el conjunto de recursos sociales inherentes a la organización social que pueden mejorar la eficiencia de la sociedad al promover una acción coordinada (Coleman, 1990), el capital social se relaciona con la creación de recursos sociales que pueden surgir de relaciones establecidas en los grupos a los que un individuo pertenece voluntariamente, por azar, por necesidad o como consecuencia de un proceso de adscripción social (Worms, 2003). En esta línea, Putman y Goss (2003, p. 14) lo conceptualizan como la mezcla de redes sociales y de normas de reciprocidad asociadas con ellas, que requiere de la cooperación entre grupos y virtudes humanas como la honestidad, el compromiso, el cumplimiento o la reciprocidad para generar valor.

La mayor carga de capital social de las cooperativas en relación con sus homónimas capitalistas, tal y como muestran los estudios de Bauer, Guzmán y Santos (2012) y Jones y Kalmi (2009), estaría respaldada en el contexto de su creación, su definición estructural y su vinculación con el territorio (Nilsson, Svendsen y Svendsen, 2012). En primer término, cabe señalar que la falta de accesibilidad a productos o servicios fue la principal razón de su creación hace más de un siglo. Basada tanto en sus particulares valores y principios como en la integración estructural de los intereses de las comunidades, la dinámica de las cooperativas ha permitido satisfacer las necesidades no cubiertas de grupos sociales, comunidades y regiones. 
Las cooperativas, en tanto organizaciones empresariales de naturaleza social, han sido utilizadas por individuos y pequeñas empresas, más o menos marginalizadas, para movilizar recursos mediante la reorganización de las relaciones entre sus actividades y el mercado (Malo y Tremblay, 2004). De esta manera, han funcionado como un instrumento de ajuste al mercado pues permiten a sus socios reducir riesgos al crear su propia empresa (Nilsson et al., 20I2).

En segundo lugar, la cooperativa se configura como una empresa en la que los socios participan plenamente en su funcionamiento productivo como consumidores y/o proveedores de bienes y servicios provocando su adscripción territorial. El modelo cooperativo permite la realización de proyectos en los cuales la población puede reconocerse y adquirir nuevas competencias confirmando una dinámica local de desarrollo (Poirier, 1996, p. 97). Además, la aplicación del principio de intercooperación, como regla específica de funcionamiento, permite la creación de un entramado empresarial propio que resulta ser un alargamiento de su propia dinámica individual resultado de la consideración de la cooperativa como una red en sí misma. Esta manera de funcionamiento permite a las cooperativas superar su limitación productiva local derivada de la vinculación territorial de los socios para operar a escala internacional, intentando resolver los efectos de un mercado cada vez más amplio (Buendía Martínez, 1999, p. 13).

\section{Desarrollo rural, políticas públicas y cooperativas}

La evolución del pensamiento científico en cuanto al desarrollo ha derivado en el enfoque multidimensional de los problemas y de las necesidades en el ámbito local. Las estrategias descendentes de tipo centralizado, basadas en el comportamiento racionalista de las décadas de los cincuenta y sesenta, dio paso a mediados de los años setenta a un enfoque de naturaleza participativa caracterizado por un alto nivel de negociación (Friedman, 1987). Posteriormente, la crisis económica de la década de los ochenta constató la incapacidad del industrialismo como base del desarrollo, debido a la eclosión de tres factores: el denominado "paso a la sociedad postindustrial", la inviabilidad de la empresa de tipo fordista y taylorista, y la creciente competencia derivada de la globalización de la economía (Hirst y Zeitlin, 1990; Vázquez Barquero, 1988, pp. II-23).

De esta forma emerge el desarrollo local como una nueva manera de abordar el desarrollo económico, dirigido a mejorar el nivel y la calidad de vida de la población. 
Considerado, desde el punto de vista económico, como un proceso de cambio y de mejora de las estructuras económicas en un territorio homogéneo, debido a sus características culturales, sociales y económicas (Comunidades Europeas, 1996), el desarrollo local o endógeno cobra en estos momentos una especial relevancia como factor clave en la reactivación económica (Jordán, Antuñano y Fuentes, 20I3).

En esta misma línea, el desarrollo rural ha pasado del paradigma de la economía dual-modernización de los años cincuenta, al enfoque territorial actual. Calificado como un proceso simultáneo de cambio institucional y productivo que se lleva a cabo en determinadas localidades y que permite avanzar en la superación de la pobreza (Schejtman y Berdegué, 2004, p. 30), es reconocido como el planteamiento adecuado para superar los desafíos cada vez complejos a los que se enfrenta el mundo rural.

La preocupación institucional por el desarrollo rural es relativamente reciente: ha pasado de ser un aspecto menor a constituir uno de los puntos prioritarios en las políticas económicas, tanto de los países occidentales como de los emergentes. Los decisores políticos son cada vez más conscientes de lo indispensable que resulta una política rural, sobre todo, por tres motivos: las considerables dificultades de las zonas rurales que debilitan la cohesión territorial de los países; el potencial económico inexplotado de las áreas rurales, y que bien conducido contribuirá a la mejora de la calidad de vida de sus habitantes y al crecimiento nacional; y el fracaso de las políticas sectoriales y de mercado para afrontar la diversidad de problemas y las potencialidades de las zonas rurales (OCDE, 2006, p. 23).

De este modo, la adopción del enfoque territorial rural por parte de las políticas públicas pivota en la promoción de las capacidades de los territorios, como función de sus recursos específicos, en la que se destaca el papel fundamental de los agentes locales y se completa con un enfoque participativo basado en estructuras asociativas y en la combinación de la lógica territorial y funcional (Maillat, 1998).

En el ámbito internacional son reconocidas las experiencias europea y canadiense, consideradas como modelos en la aplicación del enfoque de desarrollo territorial rural (Sepúlveda, Rodríguez, Echevarri y Portilla, 2003; Soto Baquero, Beduschi y Falconi, 2007). En el primer caso, el programa Leader (Liason entre Actions de Développment de l’Économie Rurale) fue lanzado en i99ı, con el objetivo de apoyar la creación y el establecimiento de grupos de acción local para la generación de proyectos empresariales en sus áreas de influencia. Reconocida como uno de los ejes básicos de la política rural de la Unión Europea (UE), la iniciativa ha conseguido dar un nuevo valor a los agentes locales y a su ubicación territorial como generadores de riqueza, en la mayoría de los casos de pequeña dimensión, 
y ha remarcado su potencialidad, lo que diferencia este programa del resto de intervenciones de carácter público (Comisión Europea, 2012; Gordo Gómez, 20II).

En el caso canadiense, el desarrollo rural ha sido una preocupación de las autoridades desde finales del siglo XIX cuando se diseñaron las primeras políticas de asentamientos. Durante la pasada centuria, las diferentes iniciativas para apoyar al mundo rural en su continuo proceso de transformación estaban incluidas en diferentes políticas sectoriales, pero fue a finales de la década de los noventa cuando se creó un departamento específico, el Secretariado Rural, lo que puso en marcha la Alianza Rural Canadiense (Partenariat Rural Canadien [PRC]) (Fairbairn, 1998).

Esta iniciativa constituye el cuadro estratégico que integra las diferentes medidas de política pública dirigida a las áreas rurales. Diseñada como un instrumento horizontal, su misión consiste en fomentar la colaboración intra e intergubernamental para garantizar que las políticas sectoriales, medidas y actividades del Gobierno nacional ofrezcan un apoyo coherente a las colectividades rurales (Goldenberg, 2008; Lennie, 2005). En los quince años de implantación del PRC, su conceptualización ha evolucionado del enfoque en el desarrollo del saber del primer PRC, al refuerzo de la colaboración intra/intercolectividades rurales del segundo. En su tercer periodo (2008-2013) fijó como objetivo la provisión de información y de instrumentos para fomentar las oportunidades locales vinculadas con determinadas ventajas competitivas (OCDE, 2010, p. 107).

La falta de un marco político integrado nacional junto con el mayor protagonismo de las autoridades provinciales, tanto por la descentralización de competencias como por la necesidad de afrontar las particularidades de la diversidad rural, ha derivado en el desarrollo de políticas rurales provinciales, aunque con diferentes grados de proactividad según el territorio.

Quebec representa un ejemplo de una política rural dinámica, no solo por su preocupación temporal, en 2014 lanzó su octavo Plan Nacional de Ruralidad (PNR), sino por su enfoque innovador. En efecto, diseñada como una política rural específica, separada de la agraria y económica tradicional, tiene como objetivo reforzar la capacidad de las colectividades y asegurar una ocupación dinámica y perdurable de las tierras rurales. Su enfoque se inscribe dentro de una visión societal de la ruralidad, compartida tanto por los diferentes niveles de la administración pública como por la sociedad civil. El desarrollo social y el reforzamiento de las capacidades colectivas son vistas como condiciones previas al desarrollo económico; mientras que la focalización sobre la ocupación dinámica 
de las tierras rurales representa una elección societal para mantener la estructura poblacional actual (Gouvernement du Québec, 2006, 2013; OCDE, 2010, p. 200).

Tanto en el ámbito nacional como en el provincial quebequense, las cooperativas tienen un papel clave en la política rural. El movimiento cooperativo tiene una gran tradición e implantación en todo el país: 6688 entidades, 17 millones de socios, I55 000 empleos directos y una cifra de negocios de 43892 millones de dólares canadienses. El análisis desagregado por provincias permite ver que en Quebec el sector tiene su máxima expresión. Con una tasa de penetración cooperativa cercana a $70 \%$, concentra $41 \%$ del total de entidades que supone una contribución al volumen de negocios cooperativo nacional de $60 \%$ (Government of Canada, 2013).

Pero, además de ser uno de sus motores económicos, su ubicación mayoritaria en las regiones rurales hace que su consolidación y desarrollo sea fundamental por tres motivos (Chambre des Communes, 20I2): como alternativa de desarrollo económico para cubrir las necesidades no satisfechas de la población, en sectores donde la acción pública y privada convencionales han fracasado; como fuerza estabilizadora con una mayor resistencia a las fluctuaciones económicas, patente en la actual crisis económica donde el sector cooperativo ha mostrado mayor fortaleza y flexibilidad, no solo para mantenerse como sector empresarial alternativo sino para continuar con la creación de empleo y proporcionar bienes y servicios a sus miembros (Birchall, 20I3; Birchall y Ketilson, 2009); y con impacto en la eficacia de los mercados, como consecuencia de la puesta en común de recursos individuales, lo que permite ejercer un poder compensatorio frente a los grandes operadores con mejoras competitivas tanto en precio como en servicios. En definitiva, se puede afirmar que las cooperativas consiguen desarrollar actividades económicas indispensables para sus comunidades y son la principal institución promotora de la política rural (OCDE, 20I0).

Estos argumentos justifican el apoyo público de las autoridades a la creación de cooperativas aunque con diferentes enfoques. En el caso del Gobierno federal, la Iniciativa de Desarrollo Cooperativo (Cooperative Development Initiative [CDI]) fue puesta en marcha en 2003 , con el fin de facilitar la generación de cooperativas en determinados ámbitos considerados prioritarios: aumento del valor añadido en la agricultura, acceso a servicios de cuidado sanitario, desarrollo rural, integración de inmigrantes, desarrollo de las comunidades autóctonas y soluciones comunitarias a problemas medioambientales. Si bien la CDI ha cumplido con sus objetivos, su evaluación ha detectado que las iniciativas futuras de desarrollo cooperativo han de realizarse en colaboración con agencias de desarrollo local dada su mayor efectividad (Government of Canada, 2013). 
Este fue el enfoque que el Gobierno quebequense adoptó a mediados de la década de los ochenta con la creación de las CDR. En colaboración con el Consejo Quebequense de la Cooperación y la Mutualidad (CQCM), como organismo de integración de las diferentes federaciones cooperativas sectoriales, las CDR son las encargadas de la creación de nuevas cooperativas para mantener los servicios de proximidad en las áreas rurales, como consecuencia del envejecimiento de la población y de la necesidad de relevo empresarial (Gouvernement du Québec, 2006).

\section{El desarrollo cooperativo como objetivo de las políticas públicas rurales: el caso de las cooperativas de desarrollo regional}

El desarrollo territorial rural, en cuanto a fenómeno endógeno y emergente, enfatiza la capacidad de iniciativa empresarial como fuerza central del crecimiento económico. Sin embargo, el potencial de la actividad emprendedora rural solo puede realizarse si existe un entorno que lo facilite y que depende, en gran medida, de las iniciativas públicas (FAO, 1997). La necesidad de programas específicos que favorezcan un entorno para la generación de proyectos empresariales está basada en las limitaciones de las áreas rurales. Entre estas, se destacan (Smallbone, 2009): la insuficiencia de los mercados locales; el restringido abanico de ocupaciones laborales y la reducida dimensión de los mercados laborales; las escasas infraestructuras tanto de transporte como de comunicaciones; el acceso limitado a los servicios de información, de asesoramiento y de apoyo; y las dificultades en el acceso a financiación.

Además, en el caso de las cooperativas, sus propias características diferenciadoras como empresas democráticas, basadas en la contribución de los socios a la actividad económica y en la justicia en el reparto de los beneficios, pueden representar una barrera de entrada a futuros emprendedores. Esta limitación es difícilmente remontable cuando la opción cooperativa se integra como una alternativa más dentro de la oferta de los agentes de desarrollo empresarial, lo que provoca una clara preferencia por estructuras capitalistas convencionales más simples de entender, de crear y de mantener (Tomas Marwick \& Associates, 1998).

Esto justificaría la relación negativa existente entre la dimensión del sector cooperativo y el capital emprendedor, entendido este como aquella capacidad para emprender que el estudio de Carrasco y Buendía-Martínez (2013) ha demostrado 
para el conjunto de los países de la UE. Esta necesidad de contar con servicios de apoyo adaptados a sus particularidades derivó en la creación de las CDR en I985. Esta iniciativa pública constituye uno de los cuatro pilares básicos de la política de desarrollo cooperativo provincial (Ministère des Finances, de l'Économie et de la Recherche, 2003).

Las CDR son entidades de segundo nivel que integran al conjunto del movimiento cooperativo de una o varias regiones administrativas ${ }^{1}$ con tres finalidades: la intercooperación, mediante el establecimiento y dirección de una red integrada de cooperativas y de organizaciones representativas; el desarrollo, al ofrecer ayuda técnica específica para la creación, la expansión y el reagrupamiento de cooperativas; y la representación institucional junto con otros actores económicos y agentes de desarrollo (Boivin, 1997; Malo, Levesque y Girard, I998). En definitiva se podría afirmar que estas entidades constituyen una red autónoma de grupos de recursos técnicos únicos y especializados para las cooperativas de base, que apoyan su supervivencia y organizan nuevas cooperativas con el consiguiente impacto en el empleo de la región (Arteau y Brassard, 2008).

Consideradas como la columna vertebral del desarrollo cooperativo, su relevancia se basa en tres aspectos. En primer lugar, su carácter intersectorial y generalista se pone de manifiesto en dos sentidos: por un lado, agrupan tanto a cooperativas de base como a sus organizaciones representativas de cualquier actividad; por otro, al contrario que otras organizaciones de desarrollo, no tienen un mercado o un sector económico como objetivo, solo está fijo el estatuto jurídico de las empresas creadas. En segundo lugar, su papel de incubadoras incluye no solo la facilitación de locales y servicios, como oferta básica de este tipo de experiencias, sino que se amplía al incluir la creación del proyecto, los productos, la tecnología, la formación técnica, los sistemas de gestión, la financiación y la comercialización (Guérard, 1986). Por último, el ámbito de actuación circunscrito a una o dos regiones administrativas, lo que permite adaptar su actuación a las necesidades y las prioridades de cada territorio (Arteau y Brassard, 2008).

En funcionamiento desde hace tres décadas, las CDR han promovido un total de 2189 cooperativas en el periodo comprendido entre 1986 y 2010 , lo que supone una contribución a la generación total del sector de 70\%. Como involucra una referencia al empleo, la actividad de las CDR ha permitido generar y mantener un total de I 4808 puestos de trabajo, aunque cabe señalar que si bien la contribución al empleo

I División territorial de la provincia de Quebec. 
total provincial no es relevante, sí resultan importantes dos aspectos: la tasa de supervivencia del empleo cooperativo nuevo es de $66 \%$ para un período de ıo años, veinte puntos por encima del nivel cooperativo general (Direction des Coopératives, 1999); y el coste medio por empleo generado es inferior al de otros programas públicos (Fédération des Coopératives de Développement Régional du Québec [Fcdrq], 1999).

Cuadro l. Evolución del movimiento cooperativo y contribución de las CDR

\begin{tabular}{|c|c|c|c|c|c|c|}
\hline & 1986 & 1991 & 1996 & 2001 & 2006 & 2010 \\
\hline Población rural (\%) & 22 & 22 & 22 & 20 & 20 & 19 \\
\hline Cooperativas declarantes & 1512 & 1737 & 1826 & 2097 & $228 \mathrm{I}$ & 2334 \\
\hline - Cooperativas de consumo & II76 & $\mathrm{I} 404$ & I 422 & I47I & 1534 & 1505 \\
\hline - Cooperativas de productores & I86 & 180 & 209 & 289 & $3 \mathrm{II}$ & 285 \\
\hline - Cooperativas de trabajadores & 139 & 128 & I47 & I8I & I87 & I77 \\
\hline -Cooperativas de trabajadores accionistas & 3 & 15 & 34 & 58 & 63 & 48 \\
\hline - Cooperativas de solidaridad & 8 & IO & I4 & 98 & I 86 & 319 \\
\hline Cooperativas constituidas & 160 & I7I & 122 & $\mathrm{I} 42$ & 157 & II5 \\
\hline - Cooperativas de consumo & --- & --- & 42 & 28 & I8 & IO \\
\hline - Cooperativas de productores & --- & --- & 23 & 24 & 24 & II \\
\hline - Cooperativas de trabajadores & --- & -- & 36 & 42 & 32 & $2 \mathrm{I}$ \\
\hline -Cooperativas de trabajadores accionistas & --- & --- & 2I & I8 & 8 & 5 \\
\hline - Cooperativas de solidaridad & -- & - & $\circ$ & 30 & 75 & 68 \\
\hline Cooperativas creadas por las CDR & 24 & 20 & 42 & 102 & $\mathrm{I} 42$ & 100 \\
\hline - Cooperativas de consumo & 3 & $\circ$ & 4 & 5 & 6 & I \\
\hline - Cooperativas de productores & I & 4 & 5 & I8 & 20 & 9 \\
\hline - Cooperativas de trabajadores & I8 & 8 & 16 & 34 & 26 & II \\
\hline -Cooperativas de trabajadores accionistas & 2 & 8 & 15 & I6 & 6 & 5 \\
\hline - Cooperativas de solidaridad & 0 & 0 & 2 & 29 & 84 & 74 \\
\hline $\begin{array}{l}\text { Contribución de las CDR a la } \\
\text { formación de cooperativas (\%) }\end{array}$ & 15.0 & II.7 & 34.4 & 7I.8 & 90.4 & 87.0 \\
\hline $\begin{array}{l}\text { Empleo generado/mantenido por las } \\
\text { cooperativas creadas por las CDR }\end{array}$ & 207 & 239 & 863 & 1100 & 609 & 320 \\
\hline
\end{tabular}

FuENTE: elaboración propia con base en los datos suministrados por la Direction du Développement des Coopératives du Ministère des Finances et de l’Économie (MFEQ) de Quebec 
Tal y como se puede ver en el Cuadro i, la evolución de las CDR no ha sido homogénea: el periodo comprendido entre la segunda mitad de los años noventa y el estallido de la primera crisis del actual siglo ha sido el de mayor crecimiento, fruto tanto del apoyo financiero constante al programa como de las condiciones económicas favorables. Resulta significativo destacar que $86 \%$ de las entidades se crearon en zonas rurales y $62 \%$ en el sector terciario (Roy, 20I2).

Particular atención merecen las cooperativas de solidaridad caracterizadas por la diversificación de sus miembros (consumidores, trabajadores y de apoyo) y su orientación a las alianzas. Esta condición permite que personas con un interés común y necesidades diversificadas se integren en una misma empresa (Clément y Bouchard, 20II, p. 63). La mayoría de estas cooperativas se concentra en los servicios personales y sociales, vitales para una población rural cada vez más envejecida; de este modo se logra mejorar su calidad de vida y también se mantiene la vitalidad de los pequeños municipios, pues se reduce el éxodo hacia los centros urbanos y se conserva la calidad de los servicios básicos (Roy, 20ı2).

\section{Conclusiones}

Las zonas rurales confrontan un conjunto de problemas como resultado de las transformaciones económicas y sociales que ocurren a escala mundial desde hace tres décadas. La intervención por parte de las autoridades públicas resulta indispensable para asegurar la supervivencia de estos territorios, aunque no existe consenso ni sobre los modelos ni sobre los instrumentos o medidas más adecuados. En cualquier caso, la promoción del empresariado representa uno de los ejes de cualquier política rural dado su impacto directo en la generación de riqueza y en la movilización de los recursos autóctonos.

La convergencia teórica de las cooperativas en los procesos de desarrollo rural está basada en su configuración estructural de naturaleza colectiva, lo que les proporciona una ventaja competitiva en relación con el resto de opciones empresariales. Además, esta ventaja está basada en la inclusión como finalidad de su creación, su naturaleza democrática y su vinculación al territorio. Este último aspecto es clave dado que su identidad, el fomento de la cohesión interna, su cultura y su evolución están intrínsecamente ligados al territorio, lo que democratiza el desarrollo territorial mediante el enriquecimiento colectivo. 
La importancia de esta vinculación ha derivado en su integración dentro de las diferentes iniciativas públicas puestas en marcha por las autoridades canadienses para asegurar la vitalidad del sector rural. En el caso quebequense, las CDR representan un instrumento eficiente de desarrollo rural, con base en la generación de empresas cooperativas que tienen un considerable efecto sobre la actividad emprendedora y el empleo. Por otro lado, representan un importante instrumento de concertación, puesto que las CDR integran al propio sector cooperativo en estructuras de segundo nivel, y así controlan y orientan su propio progreso debido a que constituyen las mejores conocedoras de las necesidades de sus territorios. En definitiva, sus resultados validan la pertinencia de este tipo de modelo empresarial colectivo de intercooperación para frenar el éxodo rural y para diversificar la base económica de las economías rurales.

A pesar de que la movilización conjunta de la red de CDR y de las estructuras representativas tradicionales de las cooperativas explicaría en buena parte el éxito obtenido, es necesario considerar que esta iniciativa no resuelve la totalidad de las problemáticas generada por la multidisciplinariedad del desarrollo rural. Por tanto, es necesaria la búsqueda continua de modelos y de formas de intervención basadas en el territorio. Si bien es cierto que los resultados han permitido que la iniciativa haya perdurado durante tres décadas, con independencia de los cambios de signo político tanto en el ámbito provincial como en el federal, las intervenciones de política pública se revisan hoy en relación con el papel que el Estado debe desempeñar en la actividad económica. En efecto, la dependencia de la financiación pública plantea algunas limitaciones en cuanto a su desarrollo futuro, debido a la diversidad de programas públicos para fomentar la actividad emprendedora cuya eficiencia y resultados habrá que comparar.

\section{Referencias}

Arteau, M., Brassard, M. y Malo, M. (2005). Les secteurs et le monvement coopératif québécois: portrait et défis. Outaouais: Chaire de Recherche du Canada en Développement des Collectivités.

Arteau, M. y Brassard, M. (2008). Coopérative et développement territorial: quels liens? Cabiers de l'ARUC, (3), 30 . 
Bauer, C., Guzmán, C. y Santos, F. (2012). Social Capital as Distinctive Feature of Social Economy Firms. International Entrepreneurship Management Journal, 8(4), 437-448.

Birchall, J. y Ketilson, L. (2009). Resilence of the Cooperative Business Model in Time of Crisis. Geneva: International Labour Office.

Birchall, J. (2013). Resilence in a Downturn the Power of Financial Cooperatives. Geneva: International Labour Office.

Boivin, I. (1997). Contribution des CDR au développement régional: Bilan et prospective. Perception et vécu d'acteurs de développement socio-économique des régions: une vue d'ensemble. Working Paper 25, Institut de Recherche et d'Enseignement pour les Coopératives de l’Université de Sherbrooke.

Buendía Martínez, I. (1999). Las sociedades cooperativas en el marco de las iniciativas públicas de desarrollo rural. Un análisis del caso español. Revista de Estudios Cooperativos, (68), 75-93.

Carrasco, I. y Buendía-Martínez, I. (2013). El tamaño del sector cooperativo en la Unión Europea: una explicación desde la teoría del crecimiento económico. Revista de Economía Pública, Social y Cooperativa, (78), I25-I48.

Chambre des Communes (2012). Situation des coopératives an Canada. Ottawa: Parlement du Canada. Recuperado el iz de diciembre 20I3, de http://www.parl. gc.ca/content/hoc/Committee/4II/COOP/Reports/RP5706528/cooprpoI/ cooprpoi-f.pdf

Chaves, R. (20I2). Las políticas públicas y las cooperativas. Ekonomiaz, Revista Vasca de Economía, (79), I68-199.

Clément, M. y Bouchard, C. (20II). Taux de survie des coopératives an Québec. Québec: Gouvernement du Québec.

Coleman, J. (1990). Foundations of Social Theory. Cambridge: Harvard University Press. Comisión Europea (2012). Ex-Post Evaluation of Rural Development Programmes 2000-2006. Bruselas: Autor/Directorate General for Agriculture and Rural Development.

Comunidades Europeas (1987). Resolución sobre la contribución de las cooperativas al desarrollo regional. Diario Oficial de las Comunidades Europeas, C 246.

Comunidades Europeas (I988). Resolución sobre las cooperativas y el movimiento cooperativo en la política de desarrollo. Diario Oficial de las Comunidades Europeas, C 309.

Comunidades Europeas (1994). Resolución sobre la contribución de las cooperativas al desarrollo regional. Diario Oficial de las Comunidades Europeas, C6I/23I. 
Coque Martínez, J. (2005). Las cooperativas como factor de desarrollo en zonas desfavorecidas. Madrid: Comité Económico y Social.

Direction des Coopératives (1999). Taux de survie des entreprises coopératives an Québec. Québec: Direction des Communications, Ministère de l'Industrie et du Commerce.

Fairbairn, B. (1998). A Preliminary History of Rural Development Policy and Programmes in Canada 1945-1995. Montreal: New Rural Economy Project. Recuperado el I3 diciembre 2013, de http://nre.concordia.ca/ftprootFull/rhistory.pdf

FAO (1997). Rural Entrepreneurship through Entrepreneurship. Roma: Autor.

Favreau, L. (2009). Développement des territoires, entreprises collectives et politiques publiques: le bilan québécois de la dernière décennie. Cabiers de l'ARUC, (4), 24.

Fédération des Coopératives de Développement Régional du Québec [Fcdrq] (1999). Le résean des coopératives de développement régional du Québec. Sainte-Foy: Autor. Friedmann, J. (1987). Planning in the Public Domain: from Knowlegde to Action. New Jersey: Princeton University Press.

Fuller-Love, N., Midmore, P., Thomas, D. y Henley, A. (2006). Entrepreneurship and Rural Economic Development: a Scenario Analysis Approach. International Journal of Entrepreneurial Behaviour E- Research, I2(5), 289-305.

García Sanz, B. (1997). La sociedad rural ante el siglo XXI. Madrid: Secretaría General Técnica, Ministerio de Agricultura, Pesca y Alimentación.

Girard, J. y Brière, S. (1999). Une identité à affirmer, un espace à occuper: aperçu bistorique du monvement coopératif an Canada français. Montreal: Chaire de Coopération Guy-Bernier, UQAM, Irecus/Université de Sherbrooke.

Goldenberg, M. (2008). A Review of Rural and Regional Development Policies and Programs. Regina: Canadian Policy Research Network. Recuperado el iz de diciembre de 2013, de http://cprn.org/documents/49496_FR.pdf

Gordo Gómez, P. (20II). Las políticas territoriales de desarrollo rural de la Unión Europea: un balance de veinte años en Castilla y León. Estudios de Economía Aplicada, 29(I), I-24.

Gouvernement du Québec (2013). Politique nationale de la ruralité. 2014-2024. Quebec: Ministère des Affaires Municipales et des Régions. Recuperado el 7 de diciembre de 20I3, de http://www.mamrot.gouv.qc.ca/pub/developpement_regional/ ruralite/politique_nationale_ruralite/PNR_20I4-2024.pdf

Government of Canada (2013). Evaluation of Rural and Cooperative

Development. Report of the Office of Audit and Evalution. Recuperado 
el 2 de diciembre de 20I3, de http://www.agr.gc.ca/eng/about-us/

offices-and-locations/office-of-audit-and-evaluation/audit-and-

evaluation-reports/agriculture-and-agri-food-canada-evaluation-reports/

evaluation-of-rural-and-co-operative-development/?id=I383668836307

Grammont, H. de (2008). El concepto de nueva ruralidad. En E. Peréz, M. Farah y

H. de Grammont (Comps.), La nueva ruralidad en América Latina. Avances teóricos y evidencias empiricas (pp. 23-44). Bogotá D. C.: Editorial Pontificia Universidad Javeriana.

Guérard, J. (1986). La création de coopératives de travail par la Coopérative de Développement Régional. Cabier de Recherche, 86(27), 86-27.

Hirst, P. y Zeitlin, J. (1990). Flexible Specialization and Post-Fordism: Theory, Evidence and Policy Implications. Londres: Birkbeck Public Policy Centre, University of London. Instituto de Promoción de la Economía Social [IPES] (1996). La creación de un clima positivo y condiciones favorables para el desarrollo en América Latina. Ginebra: Organización Internacional del Trabajo.

Jones, D. y Kalmi, P. (2009). Trust, Inequality and the Size of the Cooperative Sector: Cross-Country Evidence. Annals of Public and Cooperative Economics, 80(2), 165-195. Jordán, J., Antuñano, I. y Fuentes, V. (2013). Desarrollo endógeno y política anticrisis. Ciriec-España Revista de Economía Pública, Social y Cooperativa, (78), 245-263. Lennie, O. (2005). Linking Rural Policy and Regional Development in Western Canada. En New Approaches to Rural Policy. Lessons from around the World. (pp. 49-53). París: OCDE.

Maillat, D. (1998). Innovative Milieux and New Generations of Regional Policies. Entrepreneurship and Regional Development, Io(I), I-I6.

Malo, M., Levesque, B. y Girard, J. (1998). Empresas y desarrollo cooperativo: el modelo de Quebec. Ciriec-España Revista de Economía Pública, Social y Cooperativa, (28), 9-33. Malo, M. y Tremblay, B. (2004). Coopératives financières et solidarités. Finance et Bien Commun, (20), 66-73.

Ministère des Finances, de l'Économie et de la Recherche (2003). Cooperative Development Policy. Quebec: Autor.

Ministère des Affaires Municipales et des Régions (2006). Politique nationale de la ruralité 2007-2014. Quebec: Autor. Recuperado el is de diciembre de 2013, de http://www.mamrot.gouv.qc.ca/pub/developpement_regional/ruralite/ politique_nationale_ruralite/ruralite_politique.pdf 
Nilsson, J., Svendsen, G. y Svendsen, G. (2012). Are Large and Complex Agricultural Cooperatives Losing their Social Capital? Agribusiness. An International Journal, 28(2), 187-204.

North, D. y Smallbone, D. (2006). Developing Entrepreneurship and Enterprise in Europes Peripheral Rural Areas: some Issues Facing Policy Makers. European Planning Studies, I4(I), 4I-60.

Organización para la Cooperación y el Desarrollo Económicos [OCDE] (1993). What Future for our Countryside? París: Autor.

OCDE (2006). The New Rural Paradigm. Policies and Governance. París: Autor.

OCDE (2010). Examens de l'OCDE des politiques rurales. Québec, Canada. París: Autor. Petrin, T. (1997). Entrepreneurship as an Economic Force in Rural Development. En T. Petrin y A. Gannin (Eds.), Rural Development through Entrepreneurship (pp. 7-19). Roma: FAO.

Pezzini, M. (2003). Le développement rural dans les pays de l’OCDE. En P. Campagne y B. Dupuy (Coords.), Nonvelles stratégies pour un développement rural durable dans les pays méditerranéens. Montpellier: Ciheam.

Poirier, C. (1996). Coopératives de développement régional. En Y. Gasse y M.A. Bertrand, L'entrepreneuriat coopératif. Une perspective de développment (pp. 95-102). Québec: Fisher Presses Inc., Centre d’Entrepreneuriat et de PME.

Putnam, R. y Goss, K. (2003). Introducción. En R. Putnam (Ed.), El declive del capital social (pp. 7-34). Barcelona: Galaxia Gutenberg.

Roy, C. (20I2). Rapport d'évaluation. Entente de partenariat relativement au développement des coopératives liant le MDEIE et le CQCM pour les années 2007-2008 à 2009-20Io. Québec: Gouvernement du Québec.

Ruiz Rivera, M. y Delgado Campos, J. (2008). Territorio y nuevas ruralidades: un recorrido teórico sobre las relaciones campo-ciudad. Revista Eure, 34(I02), 77-95. Schejtman, A. y Berdegué, J. (2004). Desarrollo territorial rural. Santiago: Rimisp/ Centro Latinoamericano para el desarrollo rural. Recuperado el is de diciembre de 20I3, de http://www.fediap.com.ar/administracion/pdfs/Desarrollo\%20 Territorial\%20Rural\%20-\%20Centro\%20Latinoamericano\%2opara\%20el\%20 Desarrollo\%20Rural.pdf

Sepúlveda, S., Rodríguez A., Echevarri, R. y Portilla, M. (2003). El enfoque territorial del desarrollo rural. San José: Instituto Interamericano de Cooperación para la Agricultura. 
Smallbone, D. (2009). Fostering Entrepreneurship in Rural Areas. En J. Potter y A. Hofer (Eds.), Strengthening Entrepreneurship and Economic Development in East Germany: Lessons from Local Approaches (pp. 161-187). París: OCDE.

Soto Baquero, F., Beduschi, L. y Falconi, C. (Eds.) (2007). Desarrollo territorial rural. Análisis de las experiencias en Brasil, Chile y México. Santiago: FAO.

Tomas Marwick \& Associates (1998). Cadre de développement coopératif. Report presented to Secrétariar aux Coopérativas of Canada Governement. Manuscrito no publicado.

Unidad Regional de Desarrollo Agrícola y Rural Sostenible [Lcses] (2003). La nueva ruralidad en Europa y su interés para América Latina. Roma: FAO.

Vázquez Barquero, A. (1988). Desarrollo local. Una estrategia de creación de empleo. Madrid: Pirámide.

Valentinov, V. (2004). Toward a Social Capital Theory of Cooperative Organization. Journal of Cooperative Studies, 37(3), 5-20.

Worms, J. (2003). Viejos y nuevos vínculos en Francia. En R. Putnam (Ed.), El declive del capital social (pp. 273-344). Barcelona: Galaxia Gutenberg. 